\title{
HIGHLIGHTS
}

IMMUNOTHERAPY

\section{Robust responses to intraperitoneal IL-2 in ovarian cancer}

Ovarian cancer is the most lethal gynecologic cancer and although most patients respond to first-line treatment, more than $80 \%$ die of recurrent disease. Patients with platinum-resistant or platinum-refractory disease have a particularly dismal prognosis. Intraperitoneal (IP) chemotherapy has improved survival, and high-dose interleukin-2 (IL-2) has shown promising outcomes in patients with metastatic melanoma and renal-cell cancer. Highdose IL-2, however, remains controversial because this therapy is associated with extreme toxic effects such as hypotension and pulmonary edema.

Edwards and coauthors previously demonstrated that weekly intermittent IL-2 infusions were better tolerated than daily infusions. Therefore, the researchers conducted a phase II trial to assess the efficacy of weekly IP infusions of IL-2 in patients with platinum-resistant or refractory disease. They comment "Our studies with IP IL-2 in ovarian cancer demonstrate that loco-regional IL-2 in a moderate weekly dose is better tolerated and with apparent clinical efficacy. The durability of the responses is striking as it is for both renal cancer and melanoma”.

A total of 31 patients were enrolled, and all patients had previously received at least five courses of platinum or taxanebased therapy. Patients were treated with $6 \times 5 \mathrm{IU} / \mathrm{m}^{2}$ of IL- 2 . The primary end point was clinical response and immunologic measurements were also assessed. Overall, weekly IP IL-2 infusions were well tolerated. In total, 17 patients completed all 16 weeks of therapy. Complete responses were seen in four patients, a partial response in two patients and seven patients had stable disease. The median survival of the patient cohort was 2.1 years but in the six patients who responded, the median survival has not yet been reached (2-10+ years).

Evaluation of immune parameters revealed that eosinophil and lymphocyte counts were dramatically increased by the end of treatment. The researchers also noted a significant association between the early values of $\mathrm{CD}^{+} \mathrm{T}$ cells and survival $(P=0.05)$ and the changes in total $\mathrm{CD}^{+} \mathrm{T}$ cell counts and survival $(P=0.04)$.

This study demonstrates that weekly IP infusions of IL-2 are relatively well tolerated and show encouraging efficacy in this particularly difficult-to-treat population, with $55 \%$ of the cohort able to complete the full treatment course. No instances of pronounced hypotension, gastrointestinal fistulas, peritoneal fibrosis or pelvic abscesses were observed. "Our

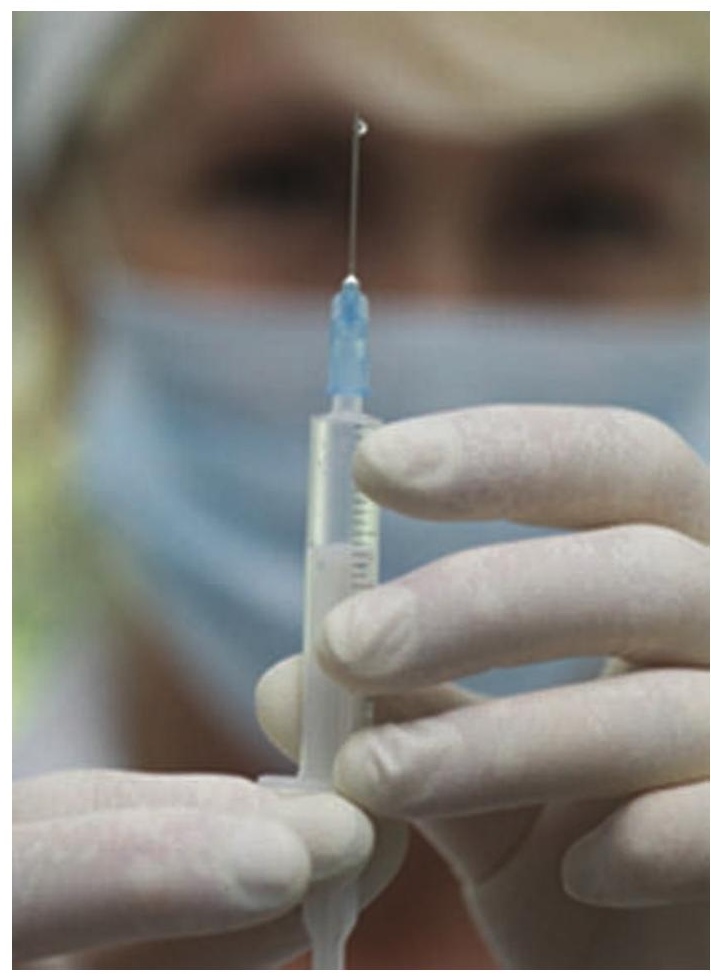

results show sufficient benefit with reasonable toxicity and call for further studies in platinum-resistant ovarian cancer patients" conclude the researchers.

\section{Lisa Hutchinson}

Original article Vlad, A. M. et al. A phase II trial of intraperitoneal interleukin- 2 in patients with platinumresistant or platinum-refractory ovarian cancer. Cancer Immunol. Immunother. 59, 293-301 (2010) 